\title{
Repensando os Referenciais Analíticos em Aprendizagem Organizacional: uma Alternativa para Análise Multidimensional
}

\author{
Roberto Ruas \\ Claudia Simone Antonello
}

Em primeiro lugar, são sempre bem-vindos os artigos que propõem uma revisão da produção científica em determinada área do conhecimento. É uma forma de os pesquisadores que ali atuam apropriarem as principais tendências, avanços e dificuldades originadas no debate recente, com visível economia de energia e tempo. Mais relevantes ainda se tornam esses trabalhos, quando aportam abrangência e qualidade. É o caso de A Produção Acadêmica sobre Aprendizagem Organizacional no Brasil, preparado por Elisabeth Loiola e Antonio Virgilio Bittencourt Bastos. Ao revisar 43 trabalhos acerca do tema publicados em três periódicos brasileiros e em anais de dois dos congressos com maior participação de pesquisadores brasileiros na área de Administração, os autores deste trabalho destacam alguns aspectos importantes do debate sobre aprendizagem organizacional no Brasil. Entre suas principais contribuições, no que concerne à caracterização de tendências e dificuldades, arrola-se o elenco a seguir.

- A concentração da produção da área em seis instituições de pósgraduação brasileiras, o que nos revela que, apesar da atratividade do tema, não se pode dizer que já decolou no Brasil (será que é um tema do tipo que decola?).

. O quase monopólio da base conceitual de origem anglo-saxônica ou, em contraparte, o pouco uso, ou baixa disponibilidade, de referências teóricas desenvolvidas por pesquisadores brasileiros.

. Na questão método, destaque para o que os autores chamam "forte orientação empírica, com base em pesquisa de desenho investigativo e relatos de experiência”. Nesta análise, os autores reforçam a perspectiva de que as metodologias com base em cortes transversais (predominantes entre os trabalhos pesquisados) não são as mais apropriadas para uma pesquisa na qual, dada a natureza "processual" da aprendizagem, a questão temporal é vital. Nesse sentido, concluem os autores que "a utilização de abordagens longitudinais, que permitem acompanhar processos e incidentes ao longo do tempo, seria mais elucidativa”. 
- Ainda no item método, os autores também destacam que a imensa maioria dos artigos é desenvolvido em nível de organização (macroprocessos) e muito pouca atenção é despendida aos microprocessos de aprendizagem, especialmente no contexto das relações de grupos. Em nosso entender, essa constatação é das mais importantes, pois a compreensão desses microprocessos de aprendizagem viabilizaria a construção de referências em torno de uma das principais questões acerca da aprendizagem organizacional: o processo de transição do individual para o coletivo.

. Finalmente, o artigo recupera um debate cuja origem, na verdade, provém da literatura internacional, também de origem anglo-saxônica, por meio do qual os autores aproveitam para fazer emergir considerações acerca da teoria e das experiências que têm marcado a discussão sobre aprendizagem organizacional no Brasil: o debate acerca da aprendizagem organizacional (AO) versus organizações de aprendizagem (OA). É a partir dessa controvérsia e das referências teóricas que sustentam esse mesmo debate, que os autores parecem construir seus principais recursos analíticos e críticos.

Entretanto, embora considerando essa démarche estimulante, até mesmo abrangente por suas possibilidades, entendemos que outros caminhos referenciais podem ser considerados. Neste sentido, recuperamos uma parte das Considerações Finais, na qual os autores justificam as dificuldades para classificar os trabalhos analisados numa linha de pensamento ou noutra (AO ou OA), considerando que "não ficaram totalmente imunes nem à subjetividade individual, nem à relativa confusão conceitual presente no campo". É por essa “relativa confusão conceitual” que iniciamos nossa contribuição mais efetiva nesse debate mediante a inclusão de outras referências.

Em primeiro lugar, é preciso reforçar a natureza “processual” da aprendizagem organizacional. Em outras palavras, é preciso contextualizá-la. Aprendizagem organizacional só passa a ter sentido em Administração se associada à mudança, haja intencionalidade ou não. Em caso contrário, pode ser tratada em outro campo de estudos e aportar uma contribuição complementar à Administração. Não é o caso. As teorias de aprendizagem organizacional estão hoje intrinsecamente vinculadas à Administração, especialmente no que diz respeito à compreensão do que pode facilitar ou dificultar os processos de mudança (do tipo novas estratégias, novos métodos e práticas de trabalho etc.). Do ponto de vista da teoria sobre mudança organizacional, o conceito de aprendizagem é valorizado por seu caráter dinâmico e integrador. Assim, os processos de aprendizagem não só valorizam a tendência à mudança contínua nas organizações, como também podem unir diferentes níveis de análise: individual, grupal e organizacional (Dodgson, 1993; Crossan et al., 1999). 
Nesse contexto, e pela própria abrangência, o debate apropria grande espectro de campos teóricos: psicológicos, sociológicos, culturais, metodológicos, além da gestão propriamente dita, é claro. Assim que tipo de referencial teórico pode auxiliar-nos a entender melhor esses processos, preservando o mais possível dessa complexidade? Certamente não será um referencial homogêneo ou unidimensional.

Por isso recorremos à configuração apresentada em Antonello (2003), a qual nos parece adequada referência conceitual, exatamente pelo fato de construir sistemáticas sem prescindir da complexidade associada à aprendizagem organizacional. Da construção da autora, vamos destacar três dos seis focos apresentados no trabalho acima citado, no interesse de contextualizar nossa argumentação e não como defesa de tese ${ }^{(1)}$. Ressalte-se que a configuração a seguir é resultado de uma busca eminentemente didático-metodológica. Isso significa que, apesar do esforço para caracterizar as especificidades de cada um dos focos, não há como negar uma grande conectividade entre eles.

\section{. Foco na Socialização da Aprendizagem Individual}

O conceito de aprendizagem é compreendido a partir de várias perspectivas, sendo principalmente desenvolvido no campo da Psicologia, ao longo de sua história evolutiva. A aplicação da aprendizagem em nível organizacional foi condicionada principalmente como socialização no plano organizacional da aprendizagem individual, treinamento e desenvolvimento (Wang e Ahmed, 2002). O processo de aprendizagem individual tem, portanto, impacto mobilizador no conceito e nas práticas de aprendizagem organizacional. Por essa razão, a relevância e, ao mesmo tempo, as dificuldades e as tensões dessa dinâmica aprendizagem individualorganizacional são tratadas em várias abordagens teóricas e constituem um dos principais elementos de referência acerca da aprendizagem organizacional em Shrivastava (1983), DeGeus (1988), Kolb (1990), Simon (1991), Kim (1993), Swieringa e Wierdsma (1995), Argyris e Schön (1996), Nonaka e Takeuchi (1997), Araujo (1998), Nonaka et al. (2000) e Probst et al. (2002).

\section{. Foco na Cultura da Organização}

Na literatura sobre aprendizagem organizacional há também forte ênfase na perspectiva cultural da organização. Cultura é apresentada como mecanismo de construção de significados - sensemaking - que guia e forma os valores, comportamentos e atitudes dos funcionários (O’Reilly e Chatman, 1996), e é por valores que os comportamentos fluem e são guiados (Simon, 1991). O processo de aprendizagem organizacional é socialmente construído (Easterby-Smith, Burgoyne e Araujo, 1999); à medida que um indivíduo interage com o ambiente, ele participa na criação da realidade, sendo compreendido como agente ativo, 
construtor da realidade na qual está inserto (Nicoline e Meznar, 1995; Gherardi et al., 1998); da mesma forma a realidade organizacional também é socialmente construída.

Assim, conceber uma empresa como comunidade de AO pressupõe que a construção do conhecimento ali vigente ocorre com base na memória coletiva organizacional, mediante adaptações progressivas de novas idéias ou propostas, partilhando os seus pressupostos e o intercâmbio de experiências. Vistos sob este ângulo, os processos de aprendizagem apresentam vínculos estreitos com a memória e a cultura da organização. A aprendizagem é o processo que exige ingressar no sistema cultural de significados (Antonello, 2003). Os membros de uma organização comunicam-se entre si e assimilam valores, normas, procedimentos, tanto na primeira socialização organizacional como durante a comunicação formal ou informal no trabalho cotidiano. Autores e obras que referenciam esse foco cultural são Simon (1991), Nicoline e Meznar (1995), Argyris e Schön (1996), Jacobson (1996), O’Reilly e Chatman (1996), Weick e Westley (1996), Gherardi et al. (1998), Easterby-Smith, Burgoyne e Araujo (1999), Richter (1999) e Bierly et al. (2000).

\section{. Foco na Gestão do Conhecimento}

A aprendizagem organizacional pode ser considerada também como processo de mudança no estado do conhecimento, implicando a sua aquisição, disseminação, refinamento, criação e implementação; como a habilidade para adquirir informação diversa e compartilhar entendimento comum, de forma que este conhecimento possa ser explorado (Fiol, 1994); e, ainda, como a habilidade para desenvolver insights, conhecimento, e associar passado e atividades futuras (Fiol e Lyles, 1985). Bierly et al. (2000, p.597) definem: "aprendizagem é o processo de unir, expandir e desenvolver dados, informação, conhecimento e saberes”. O conhecimento organizacional é armazenado em parte nos indivíduos, na forma de experiência e competências, em parte na organização, na forma de documentos, registros, regras, regulamentos e padrões etc (Weick e Roberts, 1993).

A aquisição de conhecimento está claramente relacionada com o processo de aprendizagem e é mais ampla e mais profunda do que a mera acumulação de dados e informação (Davenport e Prusak, 1998). Por isso a aprendizagem surge da tensão entre o novo e o velho conhecimento, e também da transformação do conhecimento presente na memória de um indivíduo. Este contexto de aprendizagem acontece quando conceitos, estruturas e capacidades são criados ou se desenvolvem à luz do conhecimento que é novo ao indivíduo. Considerar a relação entre conhecimento e aprendizagem também é realçar os diferentes tipos de conhecimento requeridos em situações de trabalho, bem como a diversidade de tipos e níveis de aprendizagem (Antonello, 2003). As abordagens teóricas que 
sustentam esse foco são aquelas desenvolvidas por Fiol e Lyles (1985), Garvin (1993), Schein (1993), Weick e Roberts (1993), Fiol (1994), Gephart et al. (1996), Nonaka e Takeuchi (1997), Davenport e Prusak (1998) e Bierly et al. (2000).

Em conclusão de nossa primeira contribuição, entendemos que a abordagem apresentada acima contribui para o debate acerca de referenciais teóricos que permitam pensar os processos de aprendizagem organizacional de maneira menos restrita e mais flexível. Observe-se que esse tipo de olhar, a exemplo dos três focos sintetizados anteriormente, tende a superar a controvérsia AO versus OA, à medida que cada uma dessas abordagens da aprendizagem apropria tanto as dimensões mais sociais, quanto outras mais normativas e prescritivas. Complementa essa perspectiva a constatação de que a maioria dos autores citados acima transitam de um ponto ao outro dessa controvérsia. Por outro lado, os processos de aprendizagem representativos desses focos se legitimam a partir de uma perspectiva longitudinal, tanto na dimensão temporal, quanto na dimensão ontológica da criação do conhecimento, conforme conceito de Nonaka e Takeuchi (1997), ou seja, individual, grupal, organizacional e, por sua vez, interorganizacional.

A segunda questão do artigo para a qual pretendemos contribuir é exatamente a que se refere à abordagem metodológica longitudinal e à constatação dos autores de que essa abordagem é muito pouco explorada, especialmente quando se trata de investigações no contexto de microprocessos de aprendizagem. Compreender como a aprendizagem ocorre no local de trabalho pode auxiliar a construir e propor novas formas de sustentar e nutrir processos de aprendizagem. Com isto também se está proporcionando a possibilidade de introduzir novas unidades de análise, tais como os processos de grupos ou de comunidades de prática (Lave e Wenger, 1991), suprindo a lacuna de estudos que contribuam para esclarecer o link entre aprendizagem individual e organizacional. Também se estaria contemplando o que já foi apontado por Prange (1999) como uma defasagem nas investigações sobre aprendizagem nas organizações: processos de aprendizagem referindo-se ao como, ou seja, as formas de aprendizagem organizacional.

Embora escassos, ou talvez ainda não adequadamente difundidos, já existem alguns estudos que procuram contemplar esta abordagem metodológica. Dois deles fazem parte de uma linha de pesquisa do GAP, grupo de estudos pertencente ao Programa de Pós-Graduação em Administração da Universidade Federal do Rio Grande do Sul (PPGA/UFRGS). O primeiro, já concluído (Salinas, 2001), emprega a grounded theory e investiga os impactos da difusão de princípios e conceitos de aprendizagem organizacional nas práticas de trabalho dos auditores internos de uma organização de serviços financeiros. A partir de uma estratégia orientada para o desenvolvimento de certo tipo de competências, 
entendido como estratégico para a solução de um problema crônico da organização, o trabalho acompanha, descreve, analisa e avalia, num legítimo processo de investigação longitudinal, a exploração de métodos de aprendizagem sustentados na abordagem "aprendizagem experiencial através da solução de problemas”. Além da investigação processual, foi possível mapear as principais barreiras impostas à aprendizagem, tornando-se factível aos sujeitos da aprendizagem envidarem esforços mais focados para evitar ou minimizar tais ocorrências. A relevância científica deste trabalho apresenta-se no mínimo em três aspectos:

. No fato de que ainda são escassos os experimentos que empregam intencional e explicitamente práticas de aprendizagem organizacional, menos ainda no contexto de grupos. A proposta metodológica desta intervenção pode ser consultada em Ruas (2001).

. Pela utilização da metodologia grounded theory, também pouco difundida no Brasil.

. Por conseguir compatibilizar a abordagem processual e técnica (AO e OA), identificando as barreiras que dificultam a obtenção da aprendizagem organizacional e as competências básicas requeridas para condução deste processo.

O outro estudo proposto, em fase final, busca identificar e analisar, a partir da percepção dos participantes de programas de especialização e mestrado profissional em Administração no âmbito da UFRGS, a interação dos processos formais e informais de aprendizagem e o desenvolvimento de competências gerenciais (Antonello, 2003). As modalidades consideradas foram de cursos in company e de cursos abertos. Espera-se conhecer quais e de que forma os processos organizacionais e ações de formação são capazes de gerar aprendizagem no campo da competência gerencial numa dimensão individual e coletiva. Trata-se também de um estudo longitudinal, cuja unidade de análise é o gestor que participa dos referidos cursos. Nele também se emprega a grounded theory. A relevância científica deste estudo apóia-se em pelos menos três aspectos que dizem respeito à existência de poucos estudos nacionais:

- que tentem esclarecer as relações entre o desenvolvimento de competências e os processos de aprendizagem formais e informais;

. que busquem identificar e analisar comunidades de prática;

. que procurem conhecer e analisar qual o potencial de contribuição e possíveis relações entre o desenvolvimento de projetos no campo da aprendizagem e 
competência gerencial na percepção dos pesquisados, a partir da noção de aprendizagem experiencial.

Enfim, trabalhos de abordagens metodológicas semelhantes, longitudinais e que acompanham microprocessos de aprendizagem, tendem a contribuir fortemente para o conhecimento de situações de aprendizagem organizacional em empresas brasileiras. E, sem nenhum chauvinismo, considerada a reconhecida capacidade de adaptação e mudança da empresa brasileira, sabe-se lá se estudos desse tipo não conseguem identificar e caracterizar especificidades típicas da aprendizagem local.

Finalmente, há que se considerar que as contribuições e debates acima apresentados se tornaram possíveis graças à qualidade da revisão realizada pelos autores do texto aqui debatido e à clarividência que os conduziram a destacar nele aspectos efetivamente importantes.

\section{NotA}

${ }^{1}$ Os seis focos apresentados em Antonello (2003) são: Foco na Socialização da Aprendizagem Individual, Foco em Processos/Sistemas de Aprendizagem, Foco em Cultura, Foco em Gestão do Conhecimento, Foco em Melhoria Contínua e Foco em Inovação.

\section{RefERÊnCIAS Bibliográficas}

ANTONELLO, C. S.

A metamorfose da aprendizagem organizacional: uma revisão crítica. In: RUAS, R. (Coord.). Aprendizagem organizacional e competências gerenciais: experiências e análises no contexto brasileiro. No prelo.

ARAUJO, L.

Knowing and learning as networking. Management

Learning, v. 29, n. 3, 1998.
ARGYRIS, C.;

SCHÖN, D.

Organizational learning II: theory, method, and practice. Reading, MA: Addison-Wesley, 1996.

BIERLY, P. et al.

Organizational learning, knowledge and wisdom. Journal of Organizational Change Management, v. 13, n. 6, p. 595618, 2000. 
CROSSAN, M. et al.

An organizational learning framework: from intuition to institution. The Academy of Management Review, v. 24, n. 3, p. 522-537, 1999.

DAVENPORT, T.;

PRUSAK, L.

Conhecimento empresarial. Rio de Janeiro: Campus, 1998.

DEGEUS, A. P.

Planning as learning. Harvard Business Review, p. 70-74, Mar./Apr. 1988.

\section{DODGSON, M.}

Organizational learning: a review of some literatures. Organization Studies, v. 14, n. 3, p. 375-394, 1993.

EASTERBY-SMITH, M.; BURGOYNE, J.;

ARAUJO, L. (Orgs.).

Organizational learning and the learning organization: developments in theory and practice. London: Sage Publications, 1999.

FIOL, M.

Consensus, diversity, and learning in organisations. Organisation Science, v. 5, p. 403-420, 1994.

FIOL, C. M.;

LYLES, M. A.

Organisational learning. Academy of Management Review, v. 10, n. 4, p. 803-813, 1985.
GARVIN, A.

Building a learning organization. Harvard Business Review, v. 71, n. 4, p. 78-91, 1993.

GEPHART, M. et al.

Learning organizations come alive. Training Development, p. 35-45, 1996.

GHERARDI, S. et al.

Toward a social understanding of how people learn in organizations. Management Learning, v. 29, n. 3, p. 273-297, 1998.

JACOBSON, W.

Learning, culture and learning culture. Adult Education Quarterly, v. 47, n. 1, p. 15-28, 1996.

KIM, D. H.

The link between individual and organizational learning. Sloan Management Review, v. 35, n. 1, p. 37-50, 1993.

KOLB, D. et al.

Psicologia organizacional: uma abordagem vivencial. São Paulo: Atlas, 1990.

LAVE, J.;

WENGER, E.

Situated learning: legitimate peripheral participation. Cambridge: Cambridge University Press, 1991. 
NICOLINI, D.;

MEZNAR, M. B.

The social construction of organizational learning: conceptual and practical issues in the field. Human Relations, v. 48, p. 727746, 1995.

NONAKA, I.;

TAKEUCHI, $\mathrm{H}$.

Criação de conhecimento na empresa. Rio de Janeiro: Campus, 1997.

O’REILLY, C. A.;

CHATMAN, J. A.

Culture as social control: corporations, cults, and commitment. Greenwich: JAI Press, 1996.

PRANGE, C.

Organizational learning: desperately seeking theory? In: EASTERBY-SMITH, M.; BURGOYNE, J.; ARAUJO, L. (Orgs.). Organizational learning and the learning organization: developments in theory and practice. London: Sage Publications, 1999.

PROBST, G. et al.

Gestão do conhecimento. Porto Alegre: Bookman, 2002.

RICHTER, I.

Executive learning and organizational learning: diffuse power or dormant potential. In: CONGRESS OF ORGANIZA-
TIONAL LEARNING, 1., 1999, Lancaster. Proceedings... [S.l.: s.n.], 1999.

RUAS, R.

Desenvolvimento de competências gerenciais e contribuição da aprendizagem organizacional. In: FLEURY, M. T. L.; OLIVEIRA JR, M. de M. (Orgs.). Gestão estratégica do conhecimento. São Paulo: Atlas, 2001.

SALINAS, J. L.

Impactos da aprendizagem organizacional nas práticas de auditoria interna: um estudo de caso no Banco do Brasil. Porto Alegre, 2001. Tese (Doutorado em Administração) - Programa de Pós-Graduação em Administração, Universidade Federal do Rio Grande do Sul.

SCHEIN, E.

On dialogue, culture and organisational learning. Organizational Dynamics, v. 22, n. 2, p. 40-51, 1993.

SHRIVASTAVA, P.

A typology of organizational learning systems. Journal of Management Studies, v. 20, n. 1, p. 7-28, 1983.

SIMON, H. A.

Bounded rationality and organizational learning. Organization Science, v. 2, p. 125-134, 1991. 
SWIERINGA, J.;

\section{WIERDISMA, A.}

La organización que aprende. [S.l.]: Addison-Wesley, 1995.

WANG, C. L.;

AHMED, P. K.

Learning through quality and innovation. Managerial Auditing Journal, v. 17, n. 7, 2002.

WEICK, K. E.;

ROBERTS, K. H.

Collective mind in organizations: heedful interrelation on flight decks. Administrative Science Quarterly, v. 38, p. 357-81, 1993.

WEICK, K. E.;

WESTLEY, F.

Organizational learning: affirming an oxymoron. In: CLEGG, S. R.; HARDY, C.; NORD, W. (Orgs.). Handbook of organizational studies. London: Sage Publications, 1996. 